\title{
DIVISIBILITY IN CATEGORIES OF A CLASS WHICH INCLUDES THE CATEGORY OF BINARY RELATIONS
}

\author{
by D. G. FITZ-GERALD
}

(Received 29 October, 1974)

Green's relations are essential for "co-ordinatizing" semigroups. Jacqueline Klasa, in applying cognate ideas to categories $[4,5]$, has shown that divisibility in suitably-behaved categories may be described in terms of subobjects and quotients.

Here it is shown that adjoint functors which are onto objects preserve divisibility (in a certain sense). The inclusion functor of the category of sets into the category $\mathbf{R}$ of binary relations is such a functor. A slight modification of its right adjoint allows the representation of $\mathbf{R}$ as a full subcategory in a category CSL of complete semilattice morphisms.

K. A. Zaretskii's criteria for divisibility in $\mathbf{R}$ [10, Sections 2.2 and 2.3] may thus be described in terms of subobjects and quotients in CSL, although neither $\mathbf{R}$ nor CSL meets the hypotheses used by Klasa.

The concepts of "pseudo-injective" and "pseudo-projective" sets of objects are introduced to afford an internal characterization of divisibility in categories with less restrictive factoring properties (we use bicategorical structure and sometimes less). The results are applicable to $\mathbf{R}$ as well as familiar semigroups and categories, and so yield a unified treatment of several published cases.

1. Background. We work with the basic ideas of category theory as expounded by S. Maclane [6], with the purely formal difference that we compose mappings from left to right rather than the reverse. Thus epis are our left cancellable arrows, and so on. Moreover we always regard a category as consisting of arrows only. We formally identify objects with identity arrows and write $\alpha: a \rightarrow b$ to indicate that the arrow $\alpha$ has source (left identity) $a$ and target (right identity) $b$.

The characters $\mathbf{A}, \mathbf{B}, \mathbf{C}$ will indicate arbitrary categories, while $\mathbf{S}$ and $\mathbf{R}$ will be reserved for the categories of (mappings between) sets and of relations between sets respectively. Additionally we use, for a category $\mathbf{C}$ and an arrow $\alpha$ of $\mathbf{C}$, the notation $\mathbf{C} \alpha$ for the set $\{\beta \in \mathbf{C} \mid \beta=\gamma \alpha$ for some $\gamma \in C$ - and similarly for $\alpha \mathrm{C}, \mathrm{C} \alpha \mathrm{C}$, and $\alpha \mathbf{C} \alpha$.

Let $b$ be an object (i.e. identity arrow) of the category $\mathbf{C}$. The set $D$ of monos with target $b$ may be endowed with the equivalence relation $\lambda_{b}=\{(\mu, v) \in D \times D \mid C \mu=C v\}$ of mutual right division. Each $\lambda_{b}$-class is called a subobject of $b$. Dually, a quotient of the object $b$ is an equivalence class of epis, having common source $b$, under the relation $\{(\phi, \theta) \mid \phi \mathbf{C}=\theta \mathbf{C}\}$.

For subobjects $N, N^{\prime}$ of $b$, we write $N \leqq N^{\prime}$ if $\mathbf{C} v \subseteq \mathbf{C} v^{\prime}$ for some (and hence all) $v \in N$ and $v^{\prime} \in N^{\prime}$. Dually we have an ordering on quotients of $b$, also denoted by $\leqq$. We shall use the following possible comparison between subobjects and quotients. Let $a$ be the source of a mono in the subobject $N$ and $b$ the target of an epi in the quotient $Q$. Then we write $N \sim Q$ if $a \mathbf{C} b$ contains an iso. (This does not depend on the particular $a, b$ chosen.)

We say $\mathbf{C}$ has images if for each $\alpha \in \mathbf{C}$ there are arrows $\beta, \mu$ such that $\alpha=\beta \mu, \mu$ is a mono, 
and any mono right dividing $\alpha$ also right divides $\mu$. If in addition $\beta$ is an epi, we say $C$ has epic images. If the respective dual statements hold we say $\mathbf{C}$ has [monic] coimages.

If $\mathbf{C}$ has images, then the set of monos $\mu$ satisfying the conditions of the definition constitutes a subobject, to be called $\operatorname{Im}(\alpha)$, of the target of $\alpha$; the dual term is $\operatorname{Coim}(\alpha)$, a quotient of the source of $\alpha$.

A category is said to be balanced if each of its arrows which is simultaneously an epi and a mono is in fact an iso. A category $\mathbf{C}$ is filtering if every arrow $\alpha$ of $\mathbf{C}$ is a product $\alpha=\phi \mu$, where $\phi$ is an epi and $\mu$ a mono. As in [5], we say that a category $\mathbf{C}$ is quasi-strong if each arrow $\alpha$ of $\mathbf{C}$ can be written both as $\alpha=\phi \mu^{\prime}$, where $\phi$ is left invertible and $\mu^{\prime}$ is a mono, and also as $\alpha=\phi^{\prime} \mu$, where $\phi^{\prime}$ is an epi and $\mu$ is right invertible. In this case, $\mu^{\prime} \in \operatorname{Im}(\alpha)$ and $\phi^{\prime} \in \operatorname{Coim}(\alpha)$.

Klasa has, in effect, obtained the following

Result 1 [5, Proposition I5]. Let $\mathbf{C}$ be a quasi-strong category and $\alpha, \beta \in \mathbf{C}$. Then $\alpha \in \mathbf{C} \beta$ if and only if $\operatorname{Im}(\alpha) \leqq \operatorname{Im}(\beta) ;$ and $\alpha \in \beta C$ if and only if $\operatorname{Coim}(\alpha) \leqq \operatorname{Coim}(\beta)$.

Now consider the categories $\mathbf{S}$ of mappings between sets, I of (1-1) partial functions between sets, and $V$ of linear transformations between vector spaces over a certain field, with the usual composition in each case. Each category is quasi-strong and the images and coimages of their arrows correspond to the traditional ranges and quotients. There are several published results, in effect dealing with divisibility in $\mathbf{S}, \mathbf{V}$ and $\mathbf{I}$, which are thus corollaries of Result 1. These appear respectively in Section 2.2 of the work of A. H. Clifford and G. B. Preston [1, Lemmas 2.5 and 2.6, p. 52 and Exercise 6, p. 57] and in a paper of N. R. Reilly [8, Lemma 2]. (For the first result, see also G. B. Preston [7, Lemmas 1 and 2].)

The category $\mathbf{R}$ of binary relations has arrows $\left(a, \alpha, a^{\prime}\right)$-denoted alternatively by $\alpha: a \rightarrow a^{\prime}$-where $a$ and $a^{\prime}$ are sets and $\alpha \subseteq a \times a^{\prime}$. For arrows $\alpha: a \rightarrow a^{\prime}$ and $\beta: b \rightarrow b^{\prime}$ of $\mathbf{R}$, the composite $\alpha \beta$ is defined if and only if $a^{\prime}=b$ and is then given by

We put

$$
\begin{gathered}
\alpha \beta=\left\{(x, z) \in a \times b^{\prime} \mid(x, y) \in \alpha\right. \text { and } \\
(y, z) \in \beta \text { for some } y \in b\} .
\end{gathered}
$$

$$
\alpha^{-1}=\{(x, y) \mid(y, x) \in \alpha\} ;
$$

so that $\alpha^{-1}: a^{\prime} \rightarrow a$. For $s \subseteq a$, we write

Then

$$
s \alpha=\left\{v \in a^{\prime} \mid(u, v) \in \alpha \text { for some } u \in s\right\} .
$$

$$
M_{\alpha}=\{s \alpha \mid s \subseteq a\}
$$

is a complete lattice of subsets of $a^{\prime}$ closed under arbitrary set-theoretic union, and

$$
L_{\alpha}=\left\{a|t| t \in M_{\alpha-1}\right\}
$$

is a complete lattice of subsets of $a$ closed under intersection (K. A. Zaretskii [10, Section 1.7]).

Among the elegant results of Zaretskii's study of the algebraic structure of $\mathbf{R}$ was the following 
Result 2 [10, Sections 2.2 and 2.3]. Let $\alpha: a \rightarrow a^{\prime}$ and $\beta: b \rightarrow b^{\prime}$ be arrows of $\mathbf{R}$. Then $\alpha \in \mathbf{R} \beta$ if and only if $a^{\prime}=b^{\prime}$ and $M_{\alpha} \subseteq M_{\beta}$; and $\alpha \in \beta \mathbf{R}$ if and only if $a=b$ and $L_{\alpha} \subseteq L_{\beta}$.

Despite the similarity between Results 1 and 2, the latter cannot be deduced from the former, because $\mathbf{R}$ is not quasi-strong, nor even filtering, as we shall see later in Lemma 5.

2. A representation of $\mathbf{R}$. G. B. Preston and the present author in [2] reduced divisibility in $\mathbf{R}$ to a consideration of divisibility in $\mathbf{S}$ by utilizing the functor $\boldsymbol{P}: \mathbf{R} \rightarrow \mathbf{S}$ defined as follows. For $\alpha: a \rightarrow b$ in $\mathbf{R}$, and for $x \subseteq a$, let $x(\alpha P)=\{u \in b \mid(t, u) \in \alpha$ for some $t \in x\}$, so that $\alpha P: 2^{a} \rightarrow 2^{b}$ in $\mathrm{S}$. We can thus identify $2^{a}$ with $a P$, etc. It is straightforward to verify that $P$ is indeed a functor, that it is (1-1) and that its restriction $\left.P\right|_{s}$ to the subcategory $S$ of $\mathbf{R}$ is simply the usual power-set functor of $\mathbf{S}$ to $\mathbf{S}$.

LEMMA 1. The functor $P$ has a left adjoint, namely the inclusion functor $I: \mathbf{S} \rightarrow \mathbf{R}$, but no right adjoint.

Proof. For each arrow $\alpha: a \rightarrow b$ in $\mathbf{S}$ and each $t \in a,\{t \alpha\}=\{t\} \alpha P=\{t\} \alpha I P$, so that the "singleton map" $\}_{a}: t \mapsto\{t\}$ of $a$ into $a P$ satisfies the condition $\left.\left.\alpha \cdot\right\}_{b}=\right\}_{a} \cdot \alpha I P$. This is nothing but the condition that $\}: a \mapsto\}_{a}$ be a natural transformation $\}: \mathbf{S} \rightarrow I P$ (S denoting the identity functor $\mathbf{S} \rightarrow \mathbf{S}$ ).

For the opposite aspect, $\partial_{b}=\{(x, t) \mid t \in x, x \subseteq b\}$ is a relation from $b P$ to $b$ satisfying $\beta P I$. $\partial_{b}=\partial_{a} . \beta$ for each $\beta: a \rightarrow b$ in $\mathbf{R}$, so that $\partial: b \mapsto \partial_{b}$ provides a natural transformation $\partial: P I \stackrel{\rightarrow}{ } \mathbf{R}$. Moreover $\}_{a P} . \partial_{a} P$ and $\}_{a} I . \partial_{a}$ are identities in $\mathbf{S}$ and $\mathbf{R}$ respectively, so that $\}$ and $\partial$ serve as the unit and counit of the claimed adjunction.

Suppose that $J: \mathbf{S} \rightarrow \mathbf{R}$ is a right adjoint of $P$. Then $a P \mathbf{S} b \cong a \mathbf{R} b J$ for all sets $a, b$. But if $a$ is finite and $b$ has cardinal 3 ,

$$
|a P S b|=3^{|a P|} \text { while }|a R b J|=2^{|a \times b J|} .
$$

Therefore no such right adjoint exists.

We note that the left adjoint $I$ of $\boldsymbol{P}$ has the following property. A functor $\boldsymbol{F}: \mathbf{A} \rightarrow \mathbf{B}$ is onto objects if for each object $b$ of $\mathbf{B}, b=a F$ for some object $a$ of $\mathbf{A}$. We use this in

Lemma 2. Suppose the functor $F: \mathbf{A} \rightarrow \mathbf{B}$ has a left [right] adjoint $\mathbf{G}: \mathbf{B} \rightarrow \mathbf{A}$ which is onto objects. Then for arrows $\alpha: a \rightarrow b$ and $\beta: c \rightarrow b$ in $\mathbf{A},[\alpha: a \rightarrow b$ and $\beta: a \rightarrow c$ in $\mathbf{A}], \alpha \in \mathbf{A} \beta$ if and only if $\alpha F \in \mathbf{B} . \beta F[\alpha \in \beta \mathbf{A}$ if and only if $\alpha F \in \beta F . \mathbf{B}]$.

Proof. Clearly $\alpha \in \mathbf{A} \beta$ implies $\alpha F \in \mathbf{B} . \beta F$. For the converse, suppose that $\alpha F=\gamma . \beta F$ with $\gamma \in \mathbf{B}$; then $\alpha F G=\gamma G, \beta F G$. Let $\eta$ and $\partial$ be the unit and counit of the adjunction, so that

But $a=d G$ for some object $d$ of $\mathbf{B}$ and so

$$
\begin{aligned}
a \partial . \alpha=\alpha F G . b \partial & =\gamma G . \beta F G . b \partial \\
& =\gamma G . c \partial . \beta .
\end{aligned}
$$

$$
a=d G=d \eta G . d G \partial=d \eta G . a \partial ;
$$

thus

$$
\alpha=a \alpha=d \eta G . \gamma G . c \partial . \beta \in \mathbf{A} \beta .
$$


This lemma applied to the functor $P$ yields a new proof of Result 2 .

The range of the functor $P$ is easily calculated. Let $\alpha \in \mathbf{S}$ and $\alpha: a P \rightarrow b P$. Then $\alpha \in \mathbf{R} P$ if and only if $(\cup m) \alpha=\cup\{x \alpha \mid x \in m\}$ for all $m \subseteq a P$; that is if and only if $\alpha$ preserves the structure of the power set as a complete semilattice with union as supremum (which semilattice we denote by $(a P, \cup)$ or simply by $a P)$.

So let CSL denote the category of all complete semilattice morphisms-that is, of triples $\left(l, \phi, l^{\prime}\right)$ where $l$ and $l^{\prime}$ are sets endowed with supremum maps sup $: l P \rightarrow l$ and $\sup ^{\prime}: l^{\prime} P \rightarrow l^{\prime}$, and $\phi: l \rightarrow l^{\prime}$ satisfies $(\sup m) \phi=\sup ^{\prime}\{t \phi \mid t \in m\}$ for all $m \subseteq l$. Composition in CSL is defined in the usual way. Then the proof of the next lemma is quite straightforward and will be omitted.

\section{Lemma 3. The map $T: \mathbf{R} \rightarrow \mathbf{C S L}$ defined by}

$$
T:(a, \alpha, b) \mapsto(a P, \alpha P, b P)
$$

is a (1-1) full functor of $\mathbf{R}$ into $\mathbf{C S L}$.

The category CSL is a balanced category and has epic images and monic coimages. (Such a category can be regarded as a bicategory, in the sense of Isbell [3].) Subobjects of an object in CSL are in (1-1)correspondence with the usual complete subsemilattices, and quotients are in (1-1) correspondence with the usual complete congruences. Under the first correspondence, the lattice $M_{\alpha}$ (for $\alpha \in \mathbf{R}$ ) introduced in Result 2 corresponds to $\operatorname{Im}(\alpha P$ ). To discover what $L_{\alpha}$ corresponds to, we make use of the dual nature of complete semilattices, as follows.

Let $(l$, sup) be a complete semilattice and define an operation inf $: l P \rightarrow l$ by setting, for $k \subseteq l$, inf $k=\sup \{t \in l \mid t \leqslant x$ for all $x \in k\}$. Then (l, sup, inf) becomes a complete lattice.

We may also regard $l$ as a small cocomplete category and $\alpha:(l$, sup $) \rightarrow\left(l^{\prime}\right.$, sup') in CSL as a cocontinuous functor. By the classical adjoint functor theorem [6, p. 126], $\alpha$ is the left half of a Galois connexion (i.e. an adjunction) whose unique right half, $\alpha \#$ say, is given, for $x \in l$ and $y \in l^{\prime}$, by $x \leqslant y \alpha \#$ if and only if $x \alpha \leqslant y$. Equivalently, $y \alpha \#=\sup \{x \in l \mid x \alpha=y\}$, so that the "closed" elements of $l$ are the greatest elements of the classes of the congruence $\alpha \alpha^{-1}$. Further, $\alpha \#:\left(l^{\prime}\right.$, inf $\left.^{\prime}\right) \mapsto(l$,inf $)$ in CSL since right adjoints preserve products, and $(\alpha \beta) \#=\beta \# \alpha \#$ for $\alpha, \beta \in \mathbf{C S L}$, so that the map \#: $\alpha \mapsto \alpha \#(\alpha \in \mathbf{C S L})$ is a contravariant functor \# :CSL $\rightarrow$ CSL. Because the order induced in $(l$, inf $)$ is the opposite to that in $(l$, sup $),(\alpha \#) \#=\alpha$ and thus we have proved

LEMMA 4. There is a (1-1) correspondence between the set of complete congruences on ( $l$, sup) and the set of complete subsemilattices ( $m$, inf) of $(l$, inf). Specifically the complete congruence $\pi$ corresponds to the complete semilattice $\left(\pi^{*}\right.$, inf) consisting of the greatest elements from each $\pi$-class.

Additionally, it is clear that $\pi_{1} \subseteq \pi_{2}$ if and only if $\pi_{1}{ }^{*} \supseteq \pi_{2}{ }^{*}$.

In the case of the complete semilattice $(a P, \cup)$, the complete congruence $(\alpha P)(\alpha P)^{-1}$ induced by the arrow $\alpha: a \rightarrow b$ of $\mathbf{R}$ corresponds to the intersection semilattice $L_{\alpha}$ of Result 2 . For $x(\alpha P)=y(\alpha P)$ means that, for each element $t$ of the target of $\alpha$, the set $\{t\} \alpha^{-1}$ intersects both $x$ and $y$ or else intersects neither. Thus the greatest element of an $(\alpha P)(\alpha P)^{-1}$ class is the complement of a union of sets $\{t\} \alpha^{-1}$, that is is an element of $L_{\alpha}$, and vice versa. 
Thus $L_{\alpha}$ represents Coim $(\alpha P)$. Result 1, however, applies to neither $\mathbf{R}$ nor CSL.

LEMMA 5. $\mathbf{R}$ is not filtering and CSL is not quasi-strong.

Proof. Let $a=\{1,2,3\}$ and $\alpha=\{(t, u) \in a \times a \mid t \neq u\}$. Suppose that we have a factorization $\alpha=\phi \mu$ with $\phi: a \rightarrow c$ an epi in $\mathbf{R}$ and $\mu: c \rightarrow a$ a mono in $\mathbf{R}$. Then $\phi T$ is an epi of $\mathbf{R} T$, hence onto $c P$, and $\mu T$ is a mono of $\mathbf{R} T$, hence (1-1) into $a P$. Thus the range of $\alpha P$ should be isomorphic to a power set. A small calculation shows however that $\alpha P$ actually has the five-element modular non-distributive lattice $M_{5}$ as its range. Therefore $\mathbf{R}$ is not filtering.

The same arrow $\alpha T$ factors in CSL, in an essentially unique way, through an epi $\psi: a P \rightarrow M_{5}$ and a mono $\theta: M_{5} \rightarrow a P$, and it may be verified directly (or deduced from the Corollary 2 to Lemma 7 below) that $\psi$ is not left invertible and $\theta$ is not right invertible. Thus CSL is not quasi-strong.

The question then arises-under what conditions common to S, I, V, and CSL can we prove results with the content of Result 1 ?

3. Divisibility in an appropriate class of categories. We observe that $(a P, \cup)$ is freely generated, as a complete semilattice, by its singletons-in fact that $a \mapsto(a P, U)$ is the object part of a left adjoint to the forgetful functor of CSL to $\mathbf{S}$-and so is projective in CSL. Reflecting the fact that left divisibility is more difficult to deal with than right divisibility, the dual is not true- $(a P, U)$ is not injective in CSL. It does, however, have a weaker property, which we now discuss.

Call a class of objects $E$ of the category $\mathbf{C}$ pseudo-injective if, for all $a, b, c \in E$, for each object $d$ of $\mathbf{C}$ such that $c \mathbf{C} d$ contains an epi, for each $\alpha: d \rightarrow a$ and for each mono $\mu: d \rightarrow b$, there exists $\xi: b \rightarrow a$ with $\mu \xi=\alpha$. We may depict this situation in the diagram below. Note that the object $d$ need not necessarily be in the pseudo-injective class.

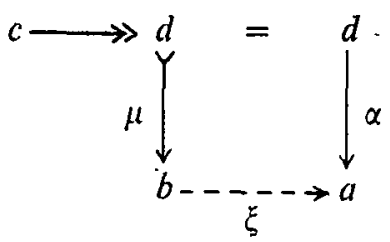

Lemma 6. $\{(b P, \cup) \mid b$ is a set $\}$ is pseudo-injective in CSL.

Proof. Suppose that $\alpha: l \rightarrow a P, \mu: l \rightarrow b P$ and $\phi: c P \rightarrow l$ are arrows of CSL with $\mu$ a mono and $\phi$ an epi, and define $\xi: b P \rightarrow a P$ as follows:

$$
\{t\} \xi=\{u \in a \mid z \in c \text { and } t \in\{z\} \phi \mu \text { imply } u \in\{z\} \phi \alpha\}
$$

for all $t \in b$, and

$$
y \xi=\cup\{\{t\} \xi \mid t \in y\}
$$

for all $y \in b P$. Then $\xi$ is in CSL and for each $z \in c,\{z\} \phi \mu \xi \subseteq\{z\} \phi \alpha$. On the other hand, if $x \notin\{z\} \phi \mu \xi$ then there exists, for each $t \in\{z\} \phi \mu, s_{t} \in c$ such that $t \in\left\{s_{t}\right\} \phi \mu$ but $x \notin\left\{s_{t}\right\} \phi \alpha$. Let $s$ 
be the collection of elements $s_{t}$; then $\{z\} \phi \mu \subseteq s \phi \mu$ but $x \notin s \phi \alpha$. Since $\mu$ is a mono, this implies that $\{z\} \phi \subseteq s \phi$ and hence that $x \notin\{z\} \phi \alpha$.

In short, this proves that $\phi \mu \xi=\phi \alpha$ and hence that $\mu \xi=\alpha$.

Of course, any subclass of a pseudo-injective class of objects is also pseudo-injective.

The term dual to "pseudo-injective" is pseudo-projective. The origin of these terms is to be found in the observation (and its dual) that if $b$ is projective, then $\{b\}$ is pseudo-projective. Moreover, if the class of all objects of a category is pseudo-projective, then each object is projective.

One can now appreciate that the following sequence of lemmas reflects the situation existing in S, I, V, and CSL. The first has the same basic content as Proposition I3 and Theorem Il of Klasa [5]. Recall that $\alpha \in \mathrm{C}$ is regular if $\alpha \in \alpha \mathrm{C} \alpha$.

Lemma 7. Let $\mathbf{C}$ be filtering and $\alpha \in \mathbf{C}$. Then $\alpha$ is regular if and only if $\operatorname{Im}(\alpha)$ exists and is $a$ retract and $\operatorname{Coim}(\alpha)$ exists and is a coretract.

Proof. There is an epi $\phi$ and a mono $\mu$ with $\alpha=\phi \mu$; so, if $\alpha$ is regular, we have $\phi \mu=\phi \mu \beta \phi \mu$ for some $\beta$. Thus $\phi=\alpha \beta \phi$ and $\mu=\mu \beta \alpha$, so that any left divisor of $\alpha$ is a left divisor of $\phi$, whence $\phi \in \operatorname{Coim}(\alpha)$. Likewise, $\mu \in \operatorname{Im}(\alpha)$. Moreover $\mu \beta \phi$ is an identity, so $\operatorname{Coim}(\alpha)$ consists of left invertible arrows, that is it is a coretract. Similarly $\operatorname{Im}(\alpha)$ is a retract.

Conversely, suppose that $\alpha=\phi \mu=\eta \beta=\gamma \theta$, where $\phi$ is an epi, $\mu$ is a mono, $\eta \in \operatorname{Coim}(\alpha)$ and is left invertible, and $\theta \in \operatorname{Im}(\alpha)$ and is right invertible. Then $\mu$ right divides $\theta$ since $\theta \in \operatorname{Im}(\alpha)$; cancelling $\mu$, one sees that $\gamma$ left divides $\phi$. Similarly $\beta$ right divides $\mu$. But $\alpha$ right divides $\beta$, hence also $\mu$, and left divides $\gamma$, hence also $\phi$. Thus $\alpha=\phi \mu \in \alpha \mathrm{C} \alpha$ and is regular.

COROLlaRy 1. If $\alpha$ is a regular element of a filtering category, then $\operatorname{Im}(\alpha) \sim \operatorname{Coim}(\alpha)$.

Proof. $\alpha=\phi \mu$ with $\mu \in \operatorname{Im}(\alpha)$ and $\phi \in \operatorname{Coim}(\alpha)$.

COROLlary 2. A complete subsemilattice of aP is a retract in CSL if and only if it is completely distributive as a complete lattice.

Proof. According to Section 3.2 of Zaretskii [10] (anticipating J.-C. Yang [9]) $\alpha \in \mathbf{R}$ is regular if and only if $M_{\alpha}$ is completely distributive. Together with Lemma 7 this gives the result.

REMARK. Elementary techniques of lattice theory can be used to provide an alternative proof of Corollary 2 and thus also an independent proof of [10, Section 3.2], referred to above.

Lemma 8. Let $\mathbf{C}$ be a balanced category which has epic images and monic coimages, and $\alpha \in$ C. Then $\operatorname{Im}(\alpha) \sim \operatorname{Coim}(\alpha)$.

Proof. Let $\theta \in \operatorname{Im}(\alpha)$ and $\phi \in \operatorname{Coim}(\alpha)$. We have $\alpha=\beta \theta=\phi \gamma$, with $\beta$ an epi and $\gamma$ a mono. Then $\theta=\delta \gamma$ and $\beta \delta=\phi$, whence $\delta$ is both an epi and a mono. Since $\mathbf{C}$ is balanced, $\delta$ is an iso from the source of $\theta$ to the target of $\phi$. 
LEMMA 9. Suppose that the balanced category $\mathbf{C}$ has images and coimages. Let $N$ be $a$ subobject of $b$ and $Q$ a quotient of a such that $N \sim Q$. Then there exists an arrow $\alpha: a \rightarrow b$ such that $\operatorname{Im}(\alpha)=N$ and $\operatorname{Coim}(\alpha)=Q$.

Proof. Let $\mu \in N$ and $\phi \in Q$; there is an iso $\theta$ for which $\phi \theta \mu$ ( $=\alpha$, say) is defined. If $\nu \in \operatorname{Im}(\alpha)$, then there exists $\lambda$ (necessarily a mono) such that $\nu=\lambda \theta \mu$. Cancel $\theta \mu$ to see that $\lambda$ right divides $\phi$; so $\lambda$ is also an epi, hence an iso. This shows that $\mu \in \operatorname{Im}(\alpha)$; similarly $\phi \in \operatorname{Coim}(\alpha)$, completing the proof.

THEOREM A. Let $\mathbf{C}$ have epic images and monic coimages. Let $\mathbf{A}$ be a full subcategory of $\mathbf{C}$ with a pseudo-projective and pseudo-injective class of objects, and suppose that $\alpha, \beta \in \mathbf{A}$. Then

(i) $\alpha \in \mathbf{A} \beta$ if and only if $\operatorname{Im}(\alpha) \leqslant \operatorname{Im}(\beta)$,

(ii) $\alpha \in \beta \mathbf{A}$ if and only if $\operatorname{Coim}(\alpha) \leqslant \operatorname{Coim}(\beta)$,

(iii) if $\mathbf{C}$ is balanced, $\alpha \in \mathbf{A} \beta \mathbf{A}$ if and only if there exist a subobject $N$ of the target of $\alpha$ and a quotient $Q$ of the source of $\beta$ such that $N \sim Q, \operatorname{Im}(\alpha) \leqslant N$ and $Q \leqslant \operatorname{Coim}(\beta)$ (and dually).

Proof. (i) If $\beta$ right divides $\alpha$, so does any mono $\mu$ in $\operatorname{Im}(\beta)$. Thus $\mu$ right divides any mono in $\operatorname{Im}(\alpha)$; that is to say, $\operatorname{Im}(\alpha) \leqslant \operatorname{Im}(\beta)$. Conversely, suppose that $\alpha=\eta \mu$ and $\beta=\theta v$ with $\mu \in \operatorname{Im}(\alpha), v \in \operatorname{Im}(\beta), \theta$ an epi and $\mu=\lambda \nu$, and consider the arrows $\eta \lambda$ and $\theta$. By the

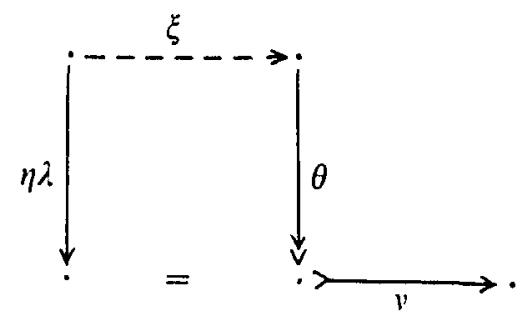

assumption of pseudo-projectiveness, there is an arrow $\xi$ satisfying $\xi \theta=\eta \lambda$. Thus $\xi \beta=\eta \lambda v=\alpha$ and moreover $\xi \in \mathbf{A}$, since $\mathbf{A}$ is full. Thus $\alpha \in \mathbf{A} \beta$.

(ii) The proof of (ii) is dual to the above.

(iii) Suppose that $\mathbf{C}$ is balanced and that $\alpha=\gamma \beta \delta(\gamma, \delta \in \mathbf{A})$. Let $N=\operatorname{Im}(\beta \delta)$ and $Q=\operatorname{Coim}(\beta \delta)$; then, by Lemma $8, N \sim Q$ while $\operatorname{Im}(\alpha) \leqslant N$ and $Q \leqslant \operatorname{Coim}(\beta)$, by parts (i) and (ii) above.

If, conversely, such $N$ and $Q$ exist, then, by Lemma 9 , there exists $\zeta$ with $\operatorname{Im}(\zeta)=N$ and $\operatorname{Coim}(\zeta)=Q ; \zeta \in \mathbf{A}$, since $\mathbf{A}$ is full. But parts (i) and (ii) above show that $\alpha \in \mathbf{A} \zeta$ and $\zeta \in \beta \mathbf{A}$; thus $\alpha \in \mathbf{A} \beta \mathbf{A}$.

This concludes the theorem.

REMARK. For balanced categories with images [coimages] the condition of pseudoprojectiveness [pseudo-injectiveness] is necessary for the statement (i) [statement (ii)] of Theorem A. 
Theorem $\mathbf{A}$ is applicable to $\mathbf{R}$ since $\mathbf{R}$ is, by Lemma 3, isomorphic to the full subcategory $\mathbf{R} T$ of CSL, and the objects of $\mathbf{R} T$ are projective and pseudo-injective in CSL (Lemma 6). This gives Result 2 cited above, and certain other results of Zaretskii [10]. In this particular case, condition (iii) of Theorem A reduces to the condition that $M_{\alpha}$ be merely order-isomorphic with some subset of $M_{\beta}[10$, Theorem 2.4]. I am unable to obtain such a neat characterization in the general case.

In the categories $\mathbf{S}, \mathbf{I}$ and $\mathbf{V}$ each object is projective and injective, so that the theorem holds, yielding once again the published results cited immediately before Result 2 above.

The category $\mathbf{P}$ of partial transformations between sets, whose arrows are functions each defined on a subset of its source, is a further example of Theorem $\mathbf{A}$. In fact, $\mathbf{P}$ is isomorphic to a full subcategory of the category $\mathbf{S}_{*}$ of pointed sets $\left[6\right.$, p. 26] under the functor $H: \mathbf{P} \rightarrow \mathbf{S}_{*}$, where, for $\delta: X \rightarrow Y$ in $\mathbf{P}, \delta H: X \cup\{X\} \rightarrow Y \cup\{Y\}$ in $\mathbf{S}_{*}$ is defined by

$$
x \delta H=\left\{\begin{array}{lr}
x \delta & \text { if } x \delta \text { is defined } \\
Y & \text { otherwise. }
\end{array}\right.
$$

This observation easily reproduces the characterization in [2] of divisibility in the category $\mathbf{P}$.

Finally, it is of interest to specialize Theorem A to provide the following characterization of Green's relations $(\mathscr{L}, \mathscr{R}, \mathscr{H}, \mathscr{D}$ and $\mathscr{J})$ on suitable semigroups of endomorphisms, such as the semigroups of endomorphisms in $\mathbf{S}, \mathbf{I}, \mathbf{V}, \mathbf{R}$ and $\mathbf{P}$.

THEOREM B. Let $\mathbf{C}$ have epic images and monic coimages. Let a be an object of $\mathbf{C}$ such that $\{a\}$ is both pseudo-projective and pseudo-injective, and suppose that $\alpha, \beta \in a \mathrm{Ca}$. Then

(i) $\alpha \mathscr{L} \beta$ if and only if $\operatorname{Im}(\alpha)=\operatorname{Im}(\beta)$,

(ii) $\alpha \mathscr{R} \beta$ if and only if $\operatorname{Coim}(\alpha)=\operatorname{Coim}(\beta)$,

(iii) $\alpha \mathscr{H} \beta$ if and only if $\operatorname{Im}(\alpha)=\operatorname{Im}(\beta)$ and $\operatorname{Coim}(\alpha)=\operatorname{Coim}(\beta)$,

(iv) if $\alpha$ and $\beta$ are regular elements, then $\alpha \mathscr{D} \beta$ if and only if $\operatorname{Im}(\alpha) \sim \operatorname{Coim}(\beta)$,

(v) if $\mathbf{C}$ is balanced, then $\alpha \mathscr{D} \beta$ if and only if $\operatorname{Im}(\alpha) \sim \operatorname{Coim}(\beta)$, and $\alpha \mathscr{J} \beta$ if and only if there exist subobjects $N, N^{\prime}$ and quotients $Q, Q^{\prime}$ such that

$$
N \sim Q, \quad N^{\prime} \sim Q^{\prime}, \quad N^{\prime} \leqslant \operatorname{Im}(\alpha) \leqslant N \text { and } Q \leqslant \operatorname{Coim}(\alpha) \leqslant Q^{\prime} .
$$

Proof. Parts (i), (ii) and (iii) are simple consequences of Theorem A, (i) and (ii).

(iv) If $\alpha$ and $\beta$ are regular, and there exists $\gamma$ such that $\alpha \mathscr{L} \gamma \mathscr{R} \beta$, then $\gamma$ is regular, and so $\operatorname{Im}(\gamma) \sim \operatorname{Coim}(\gamma)$, by Corollary 1 of Lemma 7. Thus using parts (i) and (ii) above, $\operatorname{Im}(\alpha) \sim$ $\operatorname{Coim}(\beta)$. For the converse, suppose that $\mu \in \operatorname{Im}(\alpha)$ and $\phi \in \operatorname{Coim}(\beta)$. By hypothesis, there is an iso $\theta$ such that $\phi \theta \mu$ ( $=\gamma$, say) is defined. Since $\phi$ is left invertible, $\mu \in \operatorname{Im}(\gamma)$ and it follows that $\operatorname{Im}(\gamma)=\operatorname{Im}(\alpha)$. Similarly $\operatorname{Coim}(\gamma)=\operatorname{Coim}(\beta)$ and thus $\alpha \mathscr{D} \beta$ by parts (i) and (ii) above.

(v) To characterize $\mathscr{D}$, we recall that $\operatorname{Coim}(\gamma) \sim \operatorname{Im}(\gamma)$ for $\gamma \in \mathbf{C}$, since $\mathbf{C}$ is balanced, and apply Lemma 9 and parts (i) and (ii) of this theorem. The characterization of $\mathscr{J}$ is simply an application of Theorem $\mathrm{A}$ (iii) and its dual.

ACKNOWLEDGEMENTS. The paper is condensed from parts of the author's thesis at Monash University, and it is fitting that the acknowledgements made therein be repeated here. 
My thanks go to Gordon Preston, Richard Buckdale, Bob Sullivan, Tom Hall, John and Mary Woodhams, Andrew Macfarlane, John McPhee and Brian Davey for their help.

\section{REFERENCES}

1. A. H. Clifford and G. B. Preston, The algebraic theory of semigroups, Volume 1, Amer. Math. Soc. Math. Surveys No. 7 (Providence, 1961).

2. D. G. Fitz-Gerald and G. B. Preston, Divisibility of binary relations, Bull. Austral. Math. Soc. 5 (1971), 75-86. 563-577.

3. J. R. Isbell, Some remarks concerning categories and subspaces, Can. J. Math. 9 (1957),

4. J. Klasa, Équivalences de Green dans les catégories bien filtrantes, Séminaire Dubreil-Pisot, 20 e année (1967), Fasc. 2, no. 17.

5. J. Klasa, Semisimplicity and von Neumann's regularity, Semigroup Forum 2 (1971), 354-361.

6. S. MacLane, Categories for the working mathematician, Graduate Texts in Mathematics 5 (New York, 1971).

7. G. B. Preston, Embedding any semigroup in a $\mathscr{D}$-simple semigroup, Trans. Amer. Math. Soc. 93 (1959), 351-355.

8. N. R. Reilly, Embedding inverse semigroups in bisimple inverse semigroups, Quart. J. Math. Oxford (2) 16 (1965), 183-187.

9. Jaw-Ching Yang, A theorem on the semigroup of binary relations, Proc. Amer. Math. Soc. 22 (1969), 134-135.

10. K. A. Zaretskii, Polugruppa binarnikh otnoshenii [The semigroup of binary relations], Mat. Sb. (N.S.) 61 (103) (1963), 291-305.

UNIVERSITY OF QUEENSLAND

ST. LUCIA, QUEENSLAND 